4. Mueller, B. \& Stevis-Gridneff, M. E.U. and U.K. fighting over scarce vaccines. The New York Times https://www.nytimes. com/2021/01/27/world/europe/eu-uk-covid-vaccine.html (27 January 2021)

This work was funded by Bill \& Melinda Gates Foundation grant 015897, Rockefeller Foundation grant 2020 FOD
006 and Alfred P. Sloan Foundation grant G-2019-12326,

"Innovation Information Initiative." We are grateful to Amazon Web Services for a grant from their COVID Emergency Response team for support of cloud-based computing and platform expenses. We thank Adrian Gibbs, Gilbert Faure and Marie-Christine Béné for their edits, review and constructive comments on the earlier version of the SARS-CoV-2 report prototype. The extended online version can be accessed at https://link.lens.org/ tk10f5UfAbb.

Competing interests

All authors except T.E. are employed by Cambia, a non-profit with a community-funded infrastructure that receives public and private funds. The Lens is a project of Cambia.

\title{
Democratizing biotechnology requires more than availability
}

To the Editor - In their Correspondence "The accelerating pace of biotech democratization," Jackson et al. ${ }^{1}$ find evidence for an accelerated pace at which novel biotechnologies become widely available. They state that biotechnologies that are initially available to only a handful of well-resourced specialists currently take about 4.5 years to spread to people with relatively low levels of technical skills and financial resources. By the end of the decade, the authors predict, this transition is expected to take less than 3.5 years. The authors conclude that the 'democratization' of biotechnology is accelerating.

Jackson et al. provide valuable insights into the timeframes for the spread of biotechnologies and raise interesting questions about the technical and societal factors that can explain these changes. However, the conclusion that this increased accessibility amounts to democratization of biotechnology is premature at best. The democratization of science and technology has been extensively studied by scholars in social sciences and humanities over the last thirty years ${ }^{2-4}$. Without exception, these studies conceptualize democratization not as the need to spread technologies faster and further ${ }^{5}$, but to involve society in the development and governance of technologies, from identifying issues that merit solving in the first place to deliberating the particular technologies used to solve those problems. Equating

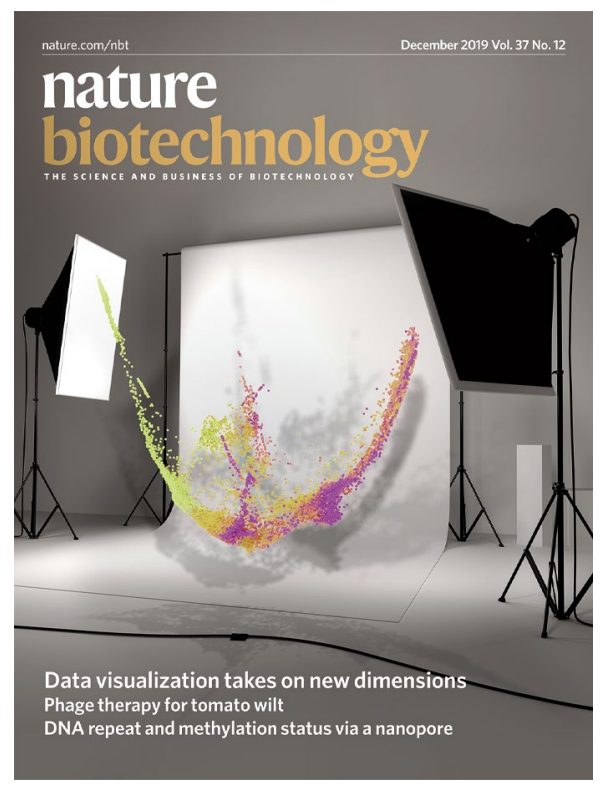

accessibility to democracy instead assumes that the technologies in question are by definition desirable and beneficial, while this is exactly what is in question in societal controversies regarding new technologies, including various biotechnologies and their applications ${ }^{6}$.

In addition, by assuming the unconditional desirability of the wide availability of biotechnologies, democratic publics are portrayed as passive recipients of scientific work. Instead, democratic theory invites us to take publics seriously: to actively listen to publics and to include their interests and concerns in the design and application of new biotechnologies ${ }^{7,8}$, so that science and society to become mutually responsive to each other ${ }^{9}$. The timeframe from first usage to widespread availability certainly matters, but the praiseworthy objective of democratizing biotechnology invites us to move beyond that.

\section{Koen Beumer (ID)}

Copernicus Institute of Sustainable DevelopmentInnovation Studies, Utrecht University, Utrecht, the Netherlands.

$凶_{e-m a i l: k . b e u m e r @ u u . n l}$

Published online: 26 March 2021

https://doi.org/10.1038/s41587-021-00890-y

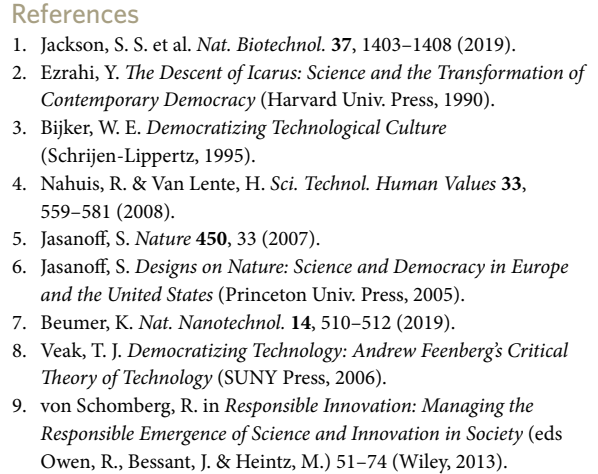

1. Jackson, S. S. et al. Nat. Biotechnol. 37, 1403-1408 (2019).

2. Ezrahi, Y. The Descent of Icarus: Science and the Transformation of Contemporary Democracy (Harvard Univ. Press, 1990).

3. Bijker, W. E. Democratizing Technological Culture (Schrijen-Lippertz, 1995).

4. Nahuis, R. \& Van Lente, H. Sci. Technol. Human Values 33, 559-581 (2008)

5. Jasanoff, S. Nature 450, 33 (2007).

6. Jasanoff, S. Designs on Nature: Science and Democracy in Europe and the United States (Princeton Univ. Press, 2005).

Beumer, K. Nat. Nanotechnol. 14, 510-512 (2019)

8. Veak, T. J. Democratizing Technology: Andrew Feenberg's Critical Theory of Technology (SUNY Press, 2006).

9. von Schomberg, R. in Responsible Innovation: Managing the Responsible Emergence of Science and Innovation in Society (eds Owen, R., Bessant, J. \& Heintz, M.) 51-74 (Wiley, 2013).

\section{Reply to: Democratizing biotechnology requires more than availability}

\author{
S. S. Jackson et al. reply - Our \\ Correspondence was written with a \\ limited definition of "democratize" in
}

mind: according to the Merriam-Webster definition, "to make (something) available to all people." That is, we were referring simply to the widespread availability of the biotechnologies studied to those with limited resources and training, and 